\title{
Hepatocellular Carcinoma with Distant Metastasis Cured by 20-Day Sorafenib Treatment
}

\author{
Seok Bae Kim \\ Department of Internal Medicine, Dankook University College of Medicine, Cheonan, South \\ Korea
}

\section{Keywords}

Hepatocellular carcinoma · Distant metastasis · Complete remission · Cure · Sorafenib

\begin{abstract}
There are only 13 cases of complete remission after sorafenib use in advanced hepatocellular carcinoma (HCC) worldwide. We herein report a rarer case in which the patient was cured after only 20 days of sorafenib use. A 61-year-old male patient was diagnosed with a huge HCC. The mass occupied almost the whole of the right hepatic lobe and a portion of segment 4. We performed extended right hepatectomy for cure. However, 3.5-cm-sized subcarinal lymph node metastasis was detected at 15 months after operation. We prescribed sorafenib $400 \mathrm{mg}$ bid for palliative treatment. The patient had severe fever, pain, and blisters on the hands and feet, so the patient stopped taking it after 20 days. Subcarinal lymph node disappeared on chest computed tomography after 3 months, and there was no evidence of recurrence for a year.
\end{abstract}

\section{Introduction}

Hepatocellular carcinoma (HCC) is the 5th most common cancer and the third common cause of death from cancer in the world. Early HCC status (single tumor $\leq 5 \mathrm{~cm}$ or 3 nodules $\leq 3$ ) is suitable for curative therapies, such as radiofrequency ablation, surgical resection, and liver transplantation. However, most patients are diagnosed at advanced stage and are not accessible to those curative treatments. However, if the patients in advanced stage took those curative treatments, they are likely to suffer from recurrence. In that case, sorafenib is now recommended as the first-line treatment in whom transarterial chemoembolization and resection are not available. 
Sorafenib is a multi-target tyrosine kinase inhibitor and can prolong the survival of HCC patients. Two randomized, double-blinded, controlled phase III clinical trials, the Sorafenib HCC Assessment Randomized Protocol (SHARP) trial, and the Asia-Pacific trials showed that sorafenib was the only effective chemotherapeutic agent. In those studies, sorafenib improved progression-free survival and enhanced the overall survival. However, it also showed many serious side effects, so early discontinuation developed. We herein report the case of a patient with distant metastasis of HCC after surgical resection that regressed following 20 days of treatment with sorafenib systemic chemotherapy.

\section{Case Presentation}

A 61-year-old male patient admitted to Dankook University Hospital for RUQ pain. He visited a local clinic before admission and had been found to have liver masses on abdominal ultrasound. We conducted 3D dynamic computed tomography (CT) to evaluate the mass, and it confirmed multiple liver masses. They were variable-sized, and the largest one was $23.1 \times 9.7 \mathrm{~cm}$. They occupied almost the whole of the right hepatic lobe and a portion of segment 4 (Fig. 1a-c). They were enhanced heterogeneously during the arterial phase and washed out during the delayed phase. The liver did not show definite surface irregularity, although there was a moderate amount of ascites in the abdominal cavity. We conducted liver-specific MRI to know that the left lobe has liver mass to decide surgical operation, and there was no more mass. On PET CT and chest CT, there was no evidence of distant metastasis (Fig. 1d). The patient was a heavy drinker who drank much alcohol every day, but he never had a liver test. The blood test conducted after hospitalization revealed a WBC of $5,440 / \mathrm{mm}^{3}, \mathrm{Hb}$ of $14.5 \mathrm{~g} / \mathrm{dL}$, platelet of $336,000 / \mathrm{mm}^{3}$, aspartate aminotransferase of $91 \mathrm{U} / \mathrm{L}$, alanine aminotransferase of $47 \mathrm{U} / \mathrm{L}$, total bilirubin of 0.57 $\mathrm{mg} / \mathrm{dL}$, albumin of $4.0 \mathrm{~g} / \mathrm{dL}$, INR of 1.1, HBsAg(-), anti-HBs(+), anti-HCV(-), and ICG R15 $12.4 \%$. The tumor marker test revealed an alpha-fetoprotein (AFP) of 2,246 ng/mL and des-gamma carboxyprothrombin of 30,652 $\mathrm{mAU} / \mathrm{mL}$. On endoscopic examination, there was no varix on the esophagus and stomach. The patient had multiple HCCs, and the mass was large. But the masses were localized in the right lobe, the segment 4 , and there was no evidence of cirrhosis, so extended right hepatectomy was performed. As a result of operation, the size of the mass was $24 \times 16 \mathrm{~cm}$, multinodular (Fig. 2). The histology showed moderately differentiated Edmondson grade III, trabecular and pseudoglandular type. The margin was free of tumor cells. Portal vein and bile duct invasion were not observed. Microvascular invasion was observed, but lymph node metastasis was not observed. The blood test performed at 3 months after surgery showed normalization to AFP $3.21 \mathrm{ng} / \mathrm{mL}$ and PIVKA $17 \mathrm{mAU} / \mathrm{mL}$. The patient was followed up by CT and blood tests every 3 months after that, and at 15 months after the operation, the AFP rose to $133.1 \mathrm{ng} / \mathrm{mL}$ and PIVKA 1,647 mAU/mL rapidly. However, on liver CT and MRI performed together, no recurrence was found, so chest CT and PET CT were performed to detect extrahepatic metastasis. A 3.5-cm-sized subcarinal lymph node was found on chest CT (Fig. 3a). The mass was detected as a hypermetabolic lesion of SULmax 6.4 on PET CT (Fig. 3b). This was diagnosed as subcarinal lymph node metastasis of HCC, and we prescribed sorafenib $400 \mathrm{mg}$ bid. The patient had severe pain and fever, and blisters on the hands and feet, so after 20 days, the patient stopped taking it. A blood test performed at 3 months after discontinuation of the drug decreased AFP to $2.91 \mathrm{ng} / \mathrm{mL}$ and PIVKA 32 $\mathrm{mAU} / \mathrm{mL}$, and the subcarinal lymph node disappeared on chest CT (Fig. 3c). After that, there was no evidence of recurrence for about a year.

\section{Karger'}



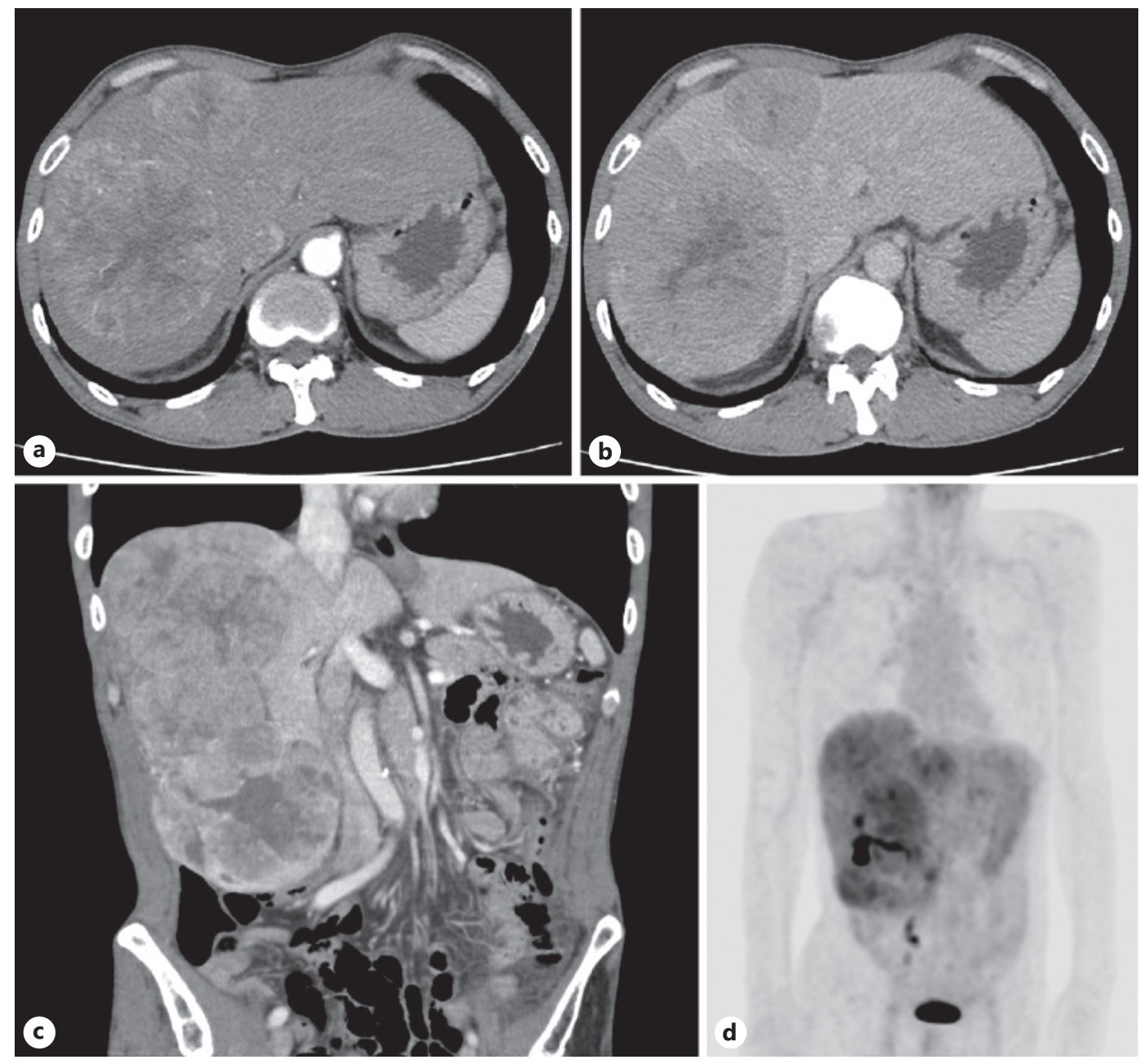

Fig. 1. Dynamic liver CT. a Arterial phase. Multiple variable-sized enhancing masses with an internal necrotic portion are found in the right hepatic lobe and segment 4 . It sized up to $10.6 \times 9.7 \mathrm{~cm}$. b Delayed phase. The masses show low density with contrast wash-out. c Coronary view. About $23.1 \times 9.7$-cm-sized huge conglomerated mass with heterogeneous density is occupying the whole liver of the right lobe. PET CT. $\mathbf{d}$ There is no evidence of distant metastasis. CT, computed tomography.

\section{Discussion}

About $80 \%$ of HCC occur in Eastern Asia and sub-Saharan Africa due to the high prevalence of chronic hepatitis B in these regions [1]. In Korea, the prevalence of chronic hepatitis B has decreased to about 3\% compared to the past, but the frequency of HCC is still ranked 5 th with $7.6 \%$ of all carcinomas. The high-risk groups of HCC are chronic viral hepatitis B and $\mathrm{C}$ and cirrhosis. Despite surveillance tests for these patients, there are still many patients diagnosed with advanced HCC. As the result, only about $20 \%$ of patients are known to receive local curative treatments such as surgical resection, liver transplantation, and radiofrequency ablation.

In general, advanced HCC is defined as a case with vascular invasion or extrahepatic metastasis according to Barcelona Clinic Liver Cancer (BCLC) classification [2]. Currently, the standard treatment for advanced HCC is sorafenib or lenvatinib, a tyrosine kinase inhibitor called systemic or targeted therapy. Both drugs can be used as the first-line drugs in Korea, 
Fig. 2. Gross finding of the mass. It sized about $24 \times 16 \mathrm{~cm}$ and showed a conglomerated multinodular type.
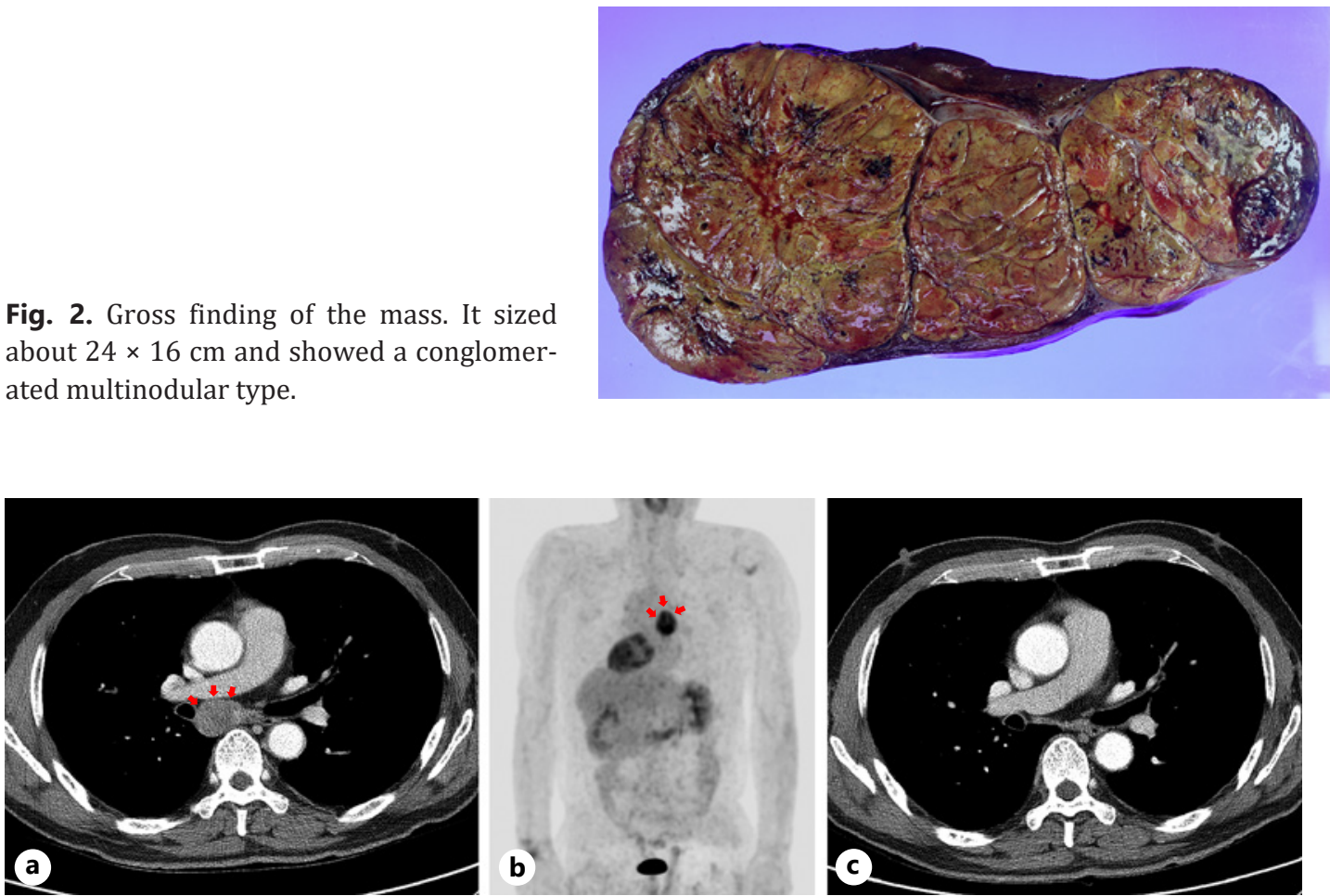

Fig. 3. Chest CT and PET CT. a Liver CT at 15 months after operation. It shows a 3.5-cm-sized new subcarinal lymph node mass indicating distant metastasis (red arrow). b The mass was detected as a hypermetabolic lesion of SULmax 6.4 on PET CT (red arrow). c Previous subcarinal lymph node disappeared after taking sorafenib for 20 days. CT, computed tomography.

but for this patient, only sorafenib should be used because lenvatinib was not yet covered by insurance in Korea at that time.

Sorafenib is a small-molecule multikinase inhibitor that inhibits Raf kinase, vascular endothelial growth factor receptor, and platelet-derived growth factor receptor. It is targeting the Raf/Mek/Erk (MAP kinase) pathway as HCC mainly overexpresses the Raf/ Mek/Erk pathway [3, 4]. Through these mechanisms, sorafenib simultaneously inhibits tumor cell proliferation and tumor angiogenesis [5, 6]. In 2 large-scale clinical trials, sorafenib prolonged survival in advanced HCCs, but no patients achieved complete remission $[7,8]$.

Since sorafenib was used in 2007, there are only 13 cases of complete remission after sorafenib use in advanced HCC worldwide [9]. Of the 13 cases, 6 patients used only sorafenib from the beginning and 4 patients underwent surgery after downstaging with sorafenib. Three patients metastasized after surgery and were cured after using sorafenib [10-19]. This present case was also treated with sorafenib after postoperative metastasis and was cured. This present case is a rarer one than 3 previous similar cases because the patient was cured after only 20 days of sorafenib use.

The most common side effects of sorafenib are diarrhea, hand-foot skin reaction (HFSR), rash, fatigue, and alopecia. Other side effects include nausea, vomiting, anorexia, and hypertension. Most of these side effects are improved by controlling or reducing the sorafenib dose. However, in the case of severe side effects such as this patient, we have to stop sorafenib. In this case, it was difficult to select the next treatment because secondary drugs such as regorafenib could not be used as insurance standards in Korea. 
Meanwhile, there are studies showing that the more severe the side effects mentioned above, the better the treatment response. As predictors that HCC responds well to sorafenib, 2 factors, which occur quickly, HFSR and AFP levels, decrease rapidly are suggested [20, 21]. So, when using sorafenib for HCC, it helps plan future treatment while observing these 2 factors. This present case is particularly suitable for the above 2 predictors. Severe HFSR occurred 20 days after taking the drug, so the drug was stopped, and AFP and PIVKA II changed to normal after 2 months. These phenomena can be said to be consistent with those in previously published studies.

In conclusion, I report a patient who had undergone surgery for a huge HCC was treated with sorafenib due to distant metastasis. However, the patient after discontinuing it after 20 days due to severe HFSR experienced complete remission persisting for more than 1 year, so I report that with a review of the literature.

\section{Statement of Ethics}

Written informed consent was obtained from the patient for publication of this case report and any accompanying images.

\section{Conflict of Interest Statement}

The author declares no conflict of interest.

\section{Funding Sources}

The author received no financial support for the research, authorship, and publication of this article.

\section{Author Contributions}

Seok Bae Kim: substantial contributions to conception and design, acquisition of data, analysis and interpretation of data, drafting the article, revising it critically for important intellectual content, and final approval of the version to be published.

\section{References}

1 Forner A, Llovet JM, Bruix J. Hepatocellular carcinoma. Lancet. 2012;379:1245-55.

2 Bruix J, Reig M, Sherman M. Evidence-based diagnosis, staging, and treatment of patients with hepatocellular carcinoma. Gastroenterology. 2016;150:835-53.

3 Cheng AL, Kang YK, Chen Z, Tsao CJ, Qin S, Kim JS, et al. Efficacy and safety of sorafenib in patients in the AsiaPacific region with advanced hepatocellular carcinoma: a phase III randomised, double-blind, placebocontrolled trial. Lancet Oncol. 2009;10:25-34.

4 So BJ, Bekaii-Saab T, Bloomston MA, Patel T. Complete clinical response of metastatic hepatocellular carcinoma to sorafenib in a patient with hemochromatosis: a case report. J Hematol Oncol. 2008;1:18.

5 Liu L, Cao Y, Chen C, Zhang X, McNabola A, Wilkie D, et al. Sorafenib blocks the RAF/MEK/ERK pathway, inhibits tumor angiogenesis, and induces tumor cell apoptosis in hepatocellular carcinoma model PLC/PRF/5. Cancer Res. 2006;66:11851-8.

6 Yoshida T, Hisamoto T, Akiba J, Koga H, Nakamura K, Tokunaga Y, et al. Spreds, inhibitors of the Ras/ERK signal transduction, are dysregulated in human hepatocellular carcinoma and linked to the malignant phenotype of tumors. Oncogene. 2006;25:6056-66. 
7 Llovet JM, Ricci S, Mazzaferro V, Hilgard P, Gane E, Blanc J-F, et al. Sorafenib in advanced hepatocellular carcinoma. N Engl J Med. 2008;359:378-90.

8 Cheng AL, Kang YK, Chen Z, Tsao CJ, Qin S, Kim JS, et al. Efficacy and safety of sorafenib in patients in the AsiaPacific region with advanced hepatocellular carcinoma: a phase III randomised, double-blind, placebocontrolled trial. Lancet Oncol. 2009;10:25-34.

9 Liu D, Liu A, Peng J, Hu Y, Feng X. Case analysis of complete remission of advanced hepatocellular carcinoma achieved with sorafenib. Eur J Med Res. 2015;20:12-7.

10 Yeganeh M, Finn RS, Saab S. Apparent remission of a solitary metastatic pulmonary lesion in a liver transplant recipient treated with sorafenib. Am J Transplant. 2009;9:2851-4.

11 Irtan S, Chopin-Laly X, Ronot M, Faivre S, Paradis V, Belghiti J. Complete regression of locally advanced hepatocellular carcinoma induced by sorafenib allowing curative resection. Liver Int. 2011;31:740-3.

12 Wang SX, Byrnes A, Verma S, Pancoast JR, Rixe O. Complete remission of unresectable hepatocellular carcinoma treated with reduced dose of sorafenib: a case report. Target Oncol. 2010;5:59-63.

13 Chelis L, Ntinos N, Souftas V, Deftereos S, Xenidis N, Chamalidou E, et al. Complete response after sorafenib therapy for hepatocellular carcinoma in an HIV-HBV co infected patient: possible synergy with HAART? A case report. Med Oncol. 2011;28(Suppl 1):S165-168.

14 Curtit E, Thiery-Vuillemin A, Nguyen T, Heyd B, Pivot X, Di Martino V, et al. Complete histologic response induced by sorafenib in advanced hepatocellular carcinoma: a case report. J Clin Oncol. 2011;29:e330-2.

15 Sacco R, Bargellini I, Gianluigi G, Bertini M, Bozzi E, Altomare E, et al. Complete response for advanced liver cancer during sorafenib therapy: case report. BMC Gastroenterol. 2011;11:4.

16 Inuzuka T, Nishikawa H, Sekikawa A, Takeda H, Henmi S, Sakamoto A, et al. Complete response of advanced hepatocellular carcinoma with multiple lung metastases treated with sorafenib: a case report. Oncology.2011; 81(Suppl 1):152-7.

17 Mizukami H, Kagawa T, Arase Y, Nakahara F, Tsuruya K, Anzai K, et al. Complete response after short-term sorafenib treatment in a patient with lymph node metastasis of hepatocellular carcinoma. Case Rep Oncol. 1912;5:380-4.

18 Lulla PD, Brammer JE, Bandeali S, Lynch GR. Sustained complete remission of metastatic hepatocellular carcinoma with single agent sorafenib. J Gastrointest Cancer. 2013;44:98-101.

19 Kim MS, Jin YJ, Lee JW, Lee JI, Kim YS, Lee SY, et al. Complete remission of advanced hepatocellular carcinoma by sorafenib: a case report. World J Gastrointest Oncol. 2013;5:38-42.

20 Vincenzi B, Santini D, Russo A, Addeo R, Giuliani F, Montella L, et al. Early skin toxicity as a predictive factor for tumor control in hepatocellular carcinoma patients treated with sorafenib. Oncologist. 2010;15:85-92.

21 Shao YY, Lin ZZ, Hsu C, Shen YC, Hsu CH, Cheng AL. Early alpha-fetoprotein response predicts treatment efficacy of antiangiogenic systemic therapy in patients with advanced hepatocellular carcinoma. Cancer. 2010; 116:4590-6. 